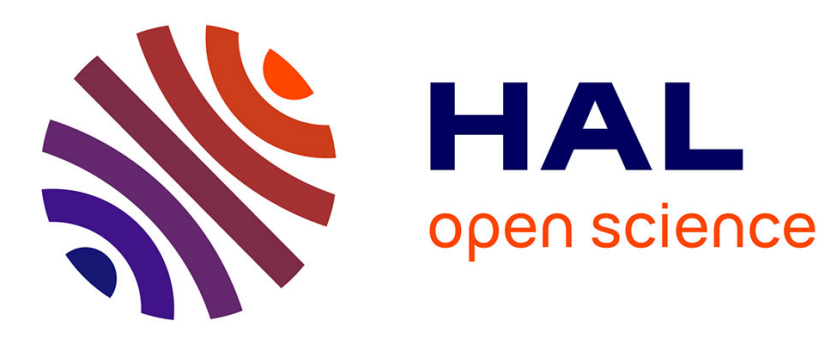

\title{
Realistic per-category pricing schemes for LTE users
} Aymen Belghith, Selem Trabelsi, Bernard Cousin

\section{To cite this version:}

Aymen Belghith, Selem Trabelsi, Bernard Cousin. Realistic per-category pricing schemes for LTE users. IEEE 12th International Symposium on Modeling and Optimization in Mobile, Ad Hoc and Wireless Networls (WIOPT), May 2014, Mammamet, Tunisia. 10.1109/WIOPT.2014.6850329 . hal01183302

\section{HAL Id: hal-01183302 \\ https://hal.science/hal-01183302}

Submitted on 7 Aug 2015

HAL is a multi-disciplinary open access archive for the deposit and dissemination of scientific research documents, whether they are published or not. The documents may come from teaching and research institutions in France or abroad, or from public or private research centers.
L'archive ouverte pluridisciplinaire HAL, est destinée au dépôt et à la diffusion de documents scientifiques de niveau recherche, publiés ou non, émanant des établissements d'enseignement et de recherche français ou étrangers, des laboratoires publics ou privés. 


\section{Realistic Per-category Pricing Schemes for LTE users}

\author{
Aymen Belghith and Selem Trabelsi \\ University of Sfax \\ Road of Aeroport Km 0.5, 3029 Sfax, Tunisia \\ Email: aymen.belghith@gmail.com \\ selem.trabelsi@gmail.com
}

\author{
Bernard Cousin \\ Université de Rennes I - IRISA \\ Campus de Beaulieu, 35042 Rennes Cedex, France \\ Email: bernard.cousin@irisa.fr
}

\begin{abstract}
As demand on data access and bandwidth explodes, service providers across cellular network infrastructures are struggling to find effective ways to manage the data traffic on their network, and meet customer expectations without eroding profitability, or attracting regulatory attention. Pricing schemes are designed to offer protable business to the operators as well as to create favorable services for the mobile subscribers. In this paper, we analyze three well-known pricing schemes proposed for Long Term Evolution (LTE) mobile networks. We then propose a realistic per-category pricing (R2P) scheme for LTE. Our pricing scheme takes into account QoS parameters, physical resources blocs utilization, user valuation, and user price categories: Gold, Silver and Bronze. Finally, we show, through extensive simulation results that our proposed pricing scheme provides the higher revenue for the operator.
\end{abstract}

\section{INTRODUCTION}

Long Term Evolution (LTE) defined by third Generation Partnership Project (3GPP) Realease 8 in 2008 is a very promising technology providing a high peak data rate of 163 Mbps in a channel bandwidth of $10 \mathrm{MHz}$ and a low latency of $15 \mathrm{~ms}$ [1]. The enhancement of LTE, called LTE-Advanced (LTE-A), aims to reach a peak data rate of $1 \mathrm{Gbps}$ in order to have a fourth generation $(4 \mathrm{G})$ access technology. This technology continues to evolve through Release 12 which is planned be completed in June 2014 [2]. This release includes advanced features such as Local Area Access (LAA) enhancement and direct device-to-device communication improvement [5].

Many kinds of applications can be simultaneously performed in an LTE User Equipment (UE) and each of them has its own requirements. For example, a Voice over Internet Protocol (VoIP) application has a strict delay and jitter requirements [3] comparing with a web browser application. However, a web application requires a very low packet loss ratio [4]. Therefore, LTE defines different QoS classes specifying their QoS Class Identifier (QCI), resources type, priority as well as delay and loss ratio thresholds. The nine standardized QCI characteristics are presented in Table 6.1.7. of [6]. Only the resource type of the first fourth QCIs is of the Guaranteed Bit Rate (GBR) type. GBR means that a minimum of bit rate resources has to be reserved. The last five QCIs resource type is the Non-GBR type.

The standard also defines user charging requirements in [7] as the charging principles for user session components and roaming. For example, real time audio and video are charged by duration of session and QoS requested and/or delivered while messaging (SMS text type) is charged by event and volume of data.
In spite of the description of the QoS classes and the charging requirements, 3 GPP does not specify the policing mechanism to be used. To the best of our knowledge, contrary to the technical aspects that were received numerous studies like scheduling, the economic aspects of radio resources have received little attention. In this paper, we study pricing in LTE.

In [8], a pricing scheme, called Fixed PRB Pricing (FPP), is proposed. For each users category, a fixed price is charged. This pricing scheme can be adapted to LTE. In fact, three kinds of LTE users are defined: Gold, Silver, and Bronze. Then, a fixed price per Physical Resource Block (PRB), which represents the resource allocation unit in LTE, is charged (see Equation (1)).

$$
P(i)=P_{\text {fixed }}(\text { Cat }(i)) \times N_{P R B}(i)
$$

where $\operatorname{Cat}(i)$ represents the category of user $i, P_{\text {fixed }}$ represents a fixed price that depends on the category of the user and $N_{P R B}(i)$ represents of the number of PRBs allocated for this user $i$.

In [9], scheme Network Load based Pricing (NLP) for $3 \mathrm{G} / 4 \mathrm{G}$ multimedia service pricing is presented. The basic idea of this scheme is to charge users with a high price when the network load becomes very important (exceeding a defined threshold). Note that this scheme were adapted to LTE network in [10] by replacing the traffic load factor $\left(L T_{-} F\right)$ by the proportion between assigned PRBs and the total number of PRBs. The price to pay is computed as follows:

$$
P(i, j)=F_{i j} \times\left(e-e^{-b * x}\right) \times L T_{-} F
$$

where:

- $\quad F_{i j}$ : is a price factor for user $i$ that has category $j$. It is constant and the same for each category when the traffic load does not exceed defined threshold $T H\left(f_{c}\right)$. Otherwise, this factor becomes variable and depends on the users category $\left(f_{j}\right)$.

- $\quad b$ : is a linearity parameter used in order to restrict the price between defined minimum and maximum price values.

- $\quad x$ : represents a QoS attribute that depends on the user QCI. When the QCI increases, $x$ decreases to charge higher price for sophisticated users. For example, when the QCI is equal to $1, x$ is equal to 9 and when the QCI becomes equal to 3, $x$ becomes equal to 7 and 
then the price paid by QCI 1 represents the highest price $\left(e-e^{-9 * b}\right.$ is the highest value).

- $\quad L T \_F$ : represents the traffic load factor.

A pricing scheme for LTE, called Subscriber Class based Pricing (SCP), is presented in [10]. This price fixed by the operator depends on the user type (Gold, Silver and Bronze) and the traffic conditions. Gold, Silver, and Bronze users are charged using (3), (4), (5), respectively.

$$
\begin{aligned}
& P(G)=\left\{\begin{array}{l}
P_{\text {fixed }}(G) \times P R B, \quad \text { if } \frac{n_{\text {ass }(G)}}{n_{\text {tot }}} \leq T H_{G} \\
\left(P_{\text {fixed }}(G)+P_{\text {add }}\right) \times P R B, \text { otherwise }
\end{array}\right. \\
& P(S)=\left\{\begin{array}{l}
P_{\text {fixed }}(S) \times P R B, \quad \text { if } \frac{n_{a s s(S)}}{n_{t o t}} \leq T H_{S} \\
\left(2 \times P_{\text {fixed }}(G)+P_{\text {add }}\right) \times P R, \text { otherwise }
\end{array}\right.
\end{aligned}
$$

$$
P(B)=\left\{\begin{array}{l}
P_{\text {fixed }}(B) \times P R B, \quad \text { if } \frac{n_{\text {ass }(B)}}{n_{\text {tot }}} \leq T H_{B} \\
\left(2 \times P_{\text {fixed }}(G)+P_{\text {fixed }}(S)+P_{\text {add }}\right) \times P R B, \quad \text { o. }
\end{array}\right.
$$

where $P_{\text {fixed }}$ represents a fixed price depending on the user type, PRB represents the number of allocated PRBs, $T H$ represents a defined threshold pointing out the congestion and is compared with the fraction between the assigned PRBs $\left(n_{\text {ass }}\right)$ and the total number of PRBs $\left(n_{t o t}\right)$, and $P_{a d d}$ represents an additional price paid when a congestion happens. $P_{a d d}$ is equal to constant factor $K$ divided by the number of the allocated PRBs.

Note that FPP does not take into account network congestion while NLP defines the same price to pay between all users when there is no congestion. In fact, each user has its willingness-to-pay (called user valuation). Moreover, the threshold that defines congestion periods is shared between all categories and therefore Bronze users, for example, can overload the network and then block Gold users because of the high price. In this case, the operator cannot profit from the high valuation of Gold users. Finally, SCP defines a very high additional price without considering that each user has its own valuation function. This function defines the price willing to pay. So, when the price requested by the operator becomes very high, the client prefers to delay/cancel his connection to the network.

In this paper, we propose a realistic per-category pricing (R2P) scheme that takes into account the user valuation, the LTE allocation unit (PRB), and the QoS classes. Moreover, it is interesting to increase the price to paid when resources become scarce and in general operators aim to take advantage of willingness-to-pay of users.

This paper is organized as follows. In Section II, we present the system model. Then, we describe our pricing models for Gold, Bronze, and Silver users in Section III. In Section IV, we present some simulation results for our pricing scheme. Conclusions and directions for future work are provided in Section V.
TABLE I. CHANNEL QUALITY AND NUMBER OF BITS TRANSMITTED PER PRB FOR VARIOUS MCSS.

\begin{tabular}{|l|l|l|}
\hline MCS & SINR interval $(\mathrm{dB})$ & Number of bits transmitted per PRB \\
\hline \hline QPSK 1/2 & {$[2.9,6.3[$} & $7 * 12 * 2 * \frac{1}{2}=84$ \\
\hline QPSK 3/4 & {$[6.3,8.6[$} & $7 * 12 * 2 * \frac{3}{4}=126$ \\
\hline 16QAM 1/2 & {$[8.6,12.7[$} & $7 * 12 * 4 * \frac{1}{2}=168$ \\
\hline 64QAM 3/4 & {$[12.7,16.9[$} & $7 * 12 * 4 * \frac{3}{4}=252$ \\
\hline 64QAM 2/3 & {$[16.9,18[$} & $7 * 12 * 6 * \frac{2}{3}=336$ \\
\hline 64QAM 3/4 & {$[18, \infty[$} & $7 * 12 * 6 * \frac{3}{4}=378$ \\
\hline
\end{tabular}

\section{System Model}

In our system model, we consider the downlink direction in the LTE network. The LTE downlink frame duration is 10 $\mathrm{ms}$ and the sub-frame duration is $1 \mathrm{~ms}$ (so there are 10 subframes for each downlink frame). Each sub-frame consists of two slots. Each slot consists of seven (respectively six) OFDM symbols when the normal (respectively extended) Cyclic Prefix (CP) is used. In the frequency domain, each resource contains 12 sub-carriers occupying a frequency of $180 \mathrm{kHz}$. In this paper, we consider a normal $\mathrm{CP}$ and therefore each resource block contains $84(7 \times 12)$ resource elements.

\section{A. User SINR and MCS}

In this paper, we consider a link adaptation mechanism that selects the Modulation and Coding Scheme used depending on the Signal-to-Interference plus Noise Ratio (SINR) of the user channel (see Table I). The SINR intervals are defined in [11]. Note that when the channel condition is better, the MCS used is more efficient and then the number of useful transmitted bits per PRB increases. For example, MCS 64QAM that represents the most efficient MCS allows station to send 378 bits per PRB when the channel state is very good (the SINR is greater than $18 \mathrm{~dB}$ ). The MCS selection is done by the evolved NodeB (eNodeB) basing on channel quality information. The eNodeB is an LTE entity which is responsible for radio transmission and reception with UEs. The evaluation of channel conditions is based on the estimated SINR of the user channel. For each PRB, effective SINR $\left(S I N R_{e f f}\right)$ is used as a metric to evaluate the channel quality information of this PRB. The SINR value of a defined sub-carrier is computed as follows:

$$
S I N R_{n}=\frac{P_{n}^{s}}{N_{0} \times W_{s c}+\sum_{i \neq s} P_{n}^{i}}
$$

where $n$ represents the index of the sub-carrier, $P^{s}$ represents the received power of the serving eNodeB, $N_{0}$ represents the noise density, and $W_{s c}$ represents the frequency spacing.

\section{B. User categories}

In this paper, we consider three categories (Gold, Silver, and Bronze) as defined in [12]. All QCIs are available for a Gold user and therefore he can use all network services such as real-time gaming and High Definition (HD) video. For a Silver user, only QCIs 3 and 5 are forbidden to use. Finally, a Bronze user is limited to use only Non-GBR services except QCI 5. The authorized QCIs and services of each users category are presented in Table II. 
TABLE II. CHARACTERISTICS OF USERS CATEGORIES.

\begin{tabular}{|c|c|c|}
\hline User category & Authorized QCIs & Examples of services \\
\hline \hline Gold & $1-9$ & IMS signaling, Conversational voice, Real-time gaming and HD video, Live video, Buffered video, Interactive gaming, TCP and P2P, etc. \\
\hline Silver & $1,2,4,6-9$ & All above except IMS signaling, real-time gaming and HD video \\
\hline Bronze & $6-9$ & Buffered video, interactive gaming, TCP and P2P \\
\hline
\end{tabular}

\section{Scheduling}

Scheduling is responsible for distributing available resources among active users in order to satisfy their QoS needs and effectively allocate radio resources in both time and frequency domains (TD and FD, respectively). In LTE, the TD scheduler is used to differentiate between users according to their categories. The user having higher priority is served first (the scheduler serves Gold, then Silver and finally Bronze users). When the TD scheduler selects a user to serve, the FD scheduler determines the best PRBs to allocate in the frequency axis. In fact, the SINRs of PRBs are variable as different frequencies are used. Therefore, the FD scheduler is responsible for selecting the most efficient PRBs in one slot.

\section{Valuation function of the user}

We now introduce the valuation functions that we propose for each users category. The estimation of the real willingnessto-pay of users is quite difficult and would need deep performance evaluation, such as in [13] for wired services. In the following we choose to take well known and often used valuation functions which represent price-related demands as in [14] and [15]. We consider that each user $i$ is willing to pay a given price $\gamma_{i}$ per transmitted rate $r_{i}$. Formally:

$$
V\left(r_{i}\right)=\gamma_{i} \times r_{i}
$$

Each user $i$ has its own $\gamma_{i}$. To evaluate our pricing scheme, we assume that $\gamma_{i}$ is randomly chosen according to a uniform random distribution on the continuous interval $\left[\Gamma_{1}^{G}, \Gamma_{2}^{G}\right],\left[\Gamma_{1}^{S}, \Gamma_{2}^{S}\right]$, and $\left.\left[\Gamma_{1}^{B}, \Gamma_{2}^{B}\right]\right)$, respectively for Gold, Silver and Bronze categories. If the price being to charge to the user is higher than its valuation, the resource allocation is rejected.

\section{E. Operators revenue}

The network operator affects the allocation of resources through pricing, and users react to these prices. The index of user $i$ belongs to $[1 . . N]$. The income of the operator is the sum of the prices paid by all users:

$$
\text { Revenue }=\sum_{i=1 . . N} P_{i}
$$

\section{Proposed Pricing Models: R2P}

We propose a dynamic pricing scheme for each users category (Gold, Silver or Bronze). Gold and Silver users are charged a fixed price per each PRB allocated in the non-congestion situation (the price unit is monetary unit per PRB: mu/PRB). An additional price will be charged when a congestion happens as radio resources become very scarce. The additional price of both categories increases when the remaining PRBs decreases. Moreover, the additional price for Gold users depends on the type of service (QoS). The higher quality of service is, the higher additional price is paid. Note that Gold and Silver users may have GBR or Non-GBR services.

As the Bronze users are restricted to Non-GBR services, we choose that this users category is charged a price that does not depend on the traffic conditions. Recall that this kind of services does not guarantee any minimum reserved rate. Moreover, the unit of this price is mu per rate reserved. However, as allocation unit is the PRB and the number of bits transmitted per PRB depends on the MCS used (see Table I), we have to take into account the channel condition of the users when defining the price to paid. The lower MCS used, the higher is the price to charge. Indeed, when the MCS is not efficient (for example QPSK 1/2), only 84 bits can be transmitted per PRB and therefore the transmission of the same quantity of information needs more PRBs than a more efficient MCS.

\section{A. Pricing model for Gold user}

Each Gold user $i$ is charged $P(G, i)$ computed as follows:

$$
P(G, i)=\left\{\begin{array}{l}
P_{\text {fixed }}(G) \times n_{P R B}(i), \quad \text { if } \frac{n_{\text {ass }(G)}}{n_{\text {tot }}} \leq T H_{G} \\
\left(P_{\text {fixed }}(G)+P_{\text {add }}(G)\right) \times n_{P R B}(i), \text { otherwise }
\end{array}\right.
$$

where:

- $\quad P_{\text {fixed }}(G)$ : represents a fixed price per PRB allocated and this price is paid when there is no congestion.

- $n_{P R B}(\mathrm{i})$ : represents the number of PRBs to allocate to user $i$.

- $n_{\text {ass }}(G)$ : represents the number of PRBs already assigned in one slot for Gold users.

- $n_{t o t}$ : represents the total number of PRBs in one slot. This number depends on the bandwidth used in the LTE system. For example, in a bandwidth of $20 \mathrm{MHz}$, $n_{\text {tot }}$ is equal to 100 .

- $T H_{G}$ : represents a defined threshold specifying the congestion periods for Gold users.

- $\quad P_{a d d}(G)$ : represents an additional price per PRB paid when the network is overloaded. It depends on the QoS class and the traffic condition (see (10)).

$$
P_{\text {add }}(G)=\frac{\exp ^{\beta+\frac{1}{x}}}{\operatorname{rem}_{P R B}}
$$

where:

- $\quad x$ : represents the QCI of the user. Its value is between 1 and 9. Higher QCIs for Non-GBR services are 
charged lower price. For example, when the QCI is equal to 9 , the extra price will be $\frac{e x p^{\beta+\frac{1}{9}}}{r e m_{P R B}}$ and this price is lower than that paid by a user having a QCI equal to $3\left(\frac{e x p^{\beta+\frac{1}{3}}}{r e m_{P R B}}\right)$.

- $\quad \beta$ : represents a constant coefficient. It allows the operator to adjust the additional price to paid.

- $\operatorname{rem}_{P R B}$ : represents the number of remaining PRBs $\left(\right.$ rem $m_{P R B}=n_{\text {tot }}-n_{\text {ass }}(G)$ as Gold users are served in the first step).

\section{B. Pricing model for Silver user}

Each Silver user $i$ is charged $P(S, i)$ computed as follows:

$$
P(S, i)=\left\{\begin{array}{l}
P_{\text {fixed }}(S) \times n_{P R B}(i), \quad \text { if } \frac{n_{a s s(S)}}{n_{\text {tot }}} \leq T H_{S} \\
\left(P_{\text {fixed }}(S)+P_{\text {add }}(S)\right) \times n_{P R B}(i), \text { otherwise }
\end{array}\right.
$$

The additional price defined for a Silver user is computed as follows:

$$
P_{a d d}(S)=\frac{K}{r e m_{P R B}}
$$

where $K$ is a constant factor and $r e m_{P R B}$ is the remaining PRBs after serving Gold and Silver users.

\section{Pricing model for Bronze user}

Bronze user $i$ is charged $P(B, i)$ computed as follows:

$$
P(B, i)=P_{\text {fixed }}(B) \times r(i) \times \frac{N B P_{\max }}{N B P_{i}}
$$

Where:

- $\quad r(i)$ : represents the transmitted rate delivered to user $i$.

- $\quad N B P_{\max }:$ represents the highest number of bits transmitted per one PRB (when the most efficient MCS is used, see Table I).

- $\quad N B P_{i}$ : represents the current number of bits transmitted per one PRB (for the current MCS used by user i).

\section{Simulation RESUlTS}

In this section, we present the simulation results in order to evaluate the performance of our proposed pricing model: R2P. R2P is compared with various pricing schemes: FPP, NLP, and SCP. First we present our simulation model. Then we present simulation results showing how optimal revenues are obtained from R2P. The same approach is followed for other existing pricing schemes in order to maximize the operator revenue. Finally we compare between different LTE pricing schemes.
TABLE III. MAIN SIMULATION PARAMETERS.

\begin{tabular}{|l|l|}
\hline Parameters & Values \\
\hline \hline Bandwidth & $20 \mathrm{MHz}$ \\
\hline $\begin{array}{l}\text { Total number of sub-carriers used } \\
\text { per slot }\end{array}$ & 1200 \\
\hline Number of sub-carriers per PRB & 12 \\
\hline Number of PRB available per slot & 100 \\
\hline Sub-carriers spacing & $15 \mathrm{kHz}$ \\
\hline Frame duration & $10 \mathrm{~ms}$ \\
\hline Cellular layout & 1 cell \\
\hline Call radius & $1500 \mathrm{~m}$ \\
\hline Height of eNodeB antenna & $32 \mathrm{~m}$ \\
\hline Path loss model & Cost HATA 231 \\
\hline Shadow fading & Log-normal Standard deviation $(8 \mathrm{~dB})$ \\
\hline Fast fading & Rayleigh Distribution \\
\hline Total eNodeB Transmission Power & $46 \mathrm{dBm}$ \\
\hline Thermal noise density & $-174 \mathrm{dBm} / \mathrm{Hz}$ \\
\hline Number of users & 900 \\
\hline Positions of users & Random \\
\hline MCSs of users & Selecting using link adaption \\
\hline Categories of users & Probability of $1 / 3$ for each category \\
\hline QCIs of users & Random between allowed QCIs \\
\hline Rate requested & GBR: $100 \mathrm{Kbit} / \mathrm{s}$, Non-GBR: $50 \mathrm{Kbit} / \mathrm{s}$ \\
\hline Valuations of users & Uniform \\
\hline Resource allocation & Per slot \\
\hline
\end{tabular}

\section{A. Simulation model}

We consider a system-level simulation model based on one hexagonal cell having a radius of $1500 \mathrm{~m}$. In this cell, there are 900 users. Through extensive simulations, we show that this number can congest the network. Even for lower numbers of users in the LTE system, our proposed scheme outperforms FPP, NLP and SCP. The user category, SINR, traffic characteristics, and valuation of each user are uniformly distributed.

Table III summarizes the main parameters of the systemlevel simulator we use. For example, the bandwidth used is 20 Mhz and therefore there are 100 PRBs per slot. The number of sub-carriers per PRB is 12 . Users categories are distributed uniformly between Gold, Silver and Bronze. Then, a QCI is chosen between the allowed values (see Table II). The rate requested for each user depends on the service type. Recall that a Gold/Silver user can use resource types GBR or NonGBR while a Bronze user is restricted to Non-GBR and then all Bronze users request a rate of $50 \mathrm{Kbit} / \mathrm{s}$. The valuation of each user is chosen in manner that at least a user having the most efficient MCS (64QAM 3/4) can pay requested price when there is no congestion. Finally, the scheduling decision in LTE is done each slot or each sub-frame (two slots). We select the first option.

\section{B. Revenue maximization}

In this section, we present simulation results when varying $P_{\text {fixed }}(G), P_{\text {fixed }}(S)$, and $T H_{S}$. Note that we have investigated all parameters of our proposed pricing scheme as well as of FPP, NLP, and SCP. All optimal values are presented in Table IV.

Figure 1 represents the operator revenue from Gold, Silver, and Bronze users as a function of $P_{\text {fixed }}(G)$. Note that when the price requested by the operator is high, the revenue increases while the users accept the price to charge. However, a very high price increases the blocking rate (the rate of users refusing to pay) and therefore the operator can loose customers. We show that when $P_{\text {fixed }}(G)$ increases without exceeding 
TABLE IV. OPTIMAL PARAMETERS VALUES OF DIFFERENT PRICING SCHEMES.

\begin{tabular}{|l|l|}
\hline Pricing scheme & Optimal parameters values \\
\hline \hline FPP & $\begin{array}{l}P_{\text {fixed }}(G)=11 \mathrm{mu} / \mathrm{PRB}, P_{\text {fixed }}(S)=6 \mathrm{mu} / \mathrm{PRB}, \\
\\
P_{\text {fixed }}(B)=4 \mathrm{mu} / \mathrm{PRB}\end{array}$ \\
\hline NLP & $b=1, f_{c}=2.6 \mathrm{mu}, f_{g}=0.9 \mathrm{mu}, f_{s}=0.7 \mathrm{mu}, f_{b}=1 \mathrm{mu}$, \\
& $T H=50 \%$ \\
\hline SCP & $P_{\text {fixed }}(G)=9 \mathrm{mu} / \mathrm{PRB}, T H_{G}=20 \%, P_{\text {fixed }}(S)=8$ \\
& $\mathrm{mu} / \mathrm{PRB}, K=520, T H_{S}=T H_{B}=40 \%, P_{\text {fixed }}(B)=4$ \\
& $\mathrm{mu} / \mathrm{PRB}$ \\
\hline R2P & $P_{\text {fixed }}(G)=9 \mathrm{mu} / \mathrm{PRB}, \beta=5, T H_{G}=20 \%, P_{\text {fixed }}(S)$ \\
& $=6 \mathrm{mu} / \mathrm{PRB}, K=110, T H_{S}=25 \%, P_{\text {fixed }}(B)=0.16$ \\
& $\mathrm{mu} /(\mathrm{Kbit} / \mathrm{s})$ \\
\hline
\end{tabular}

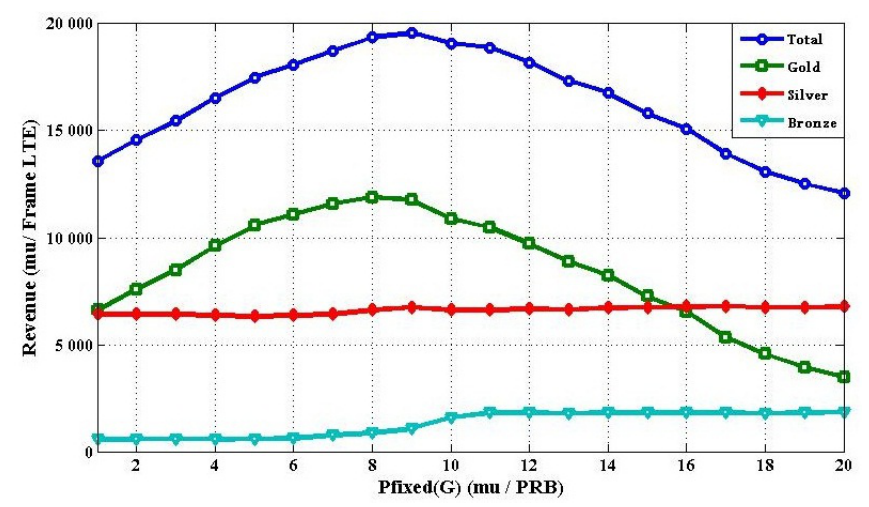

Fig. 1. Mean revenue versus $P_{\text {fixed }}(G)$ when using R2P

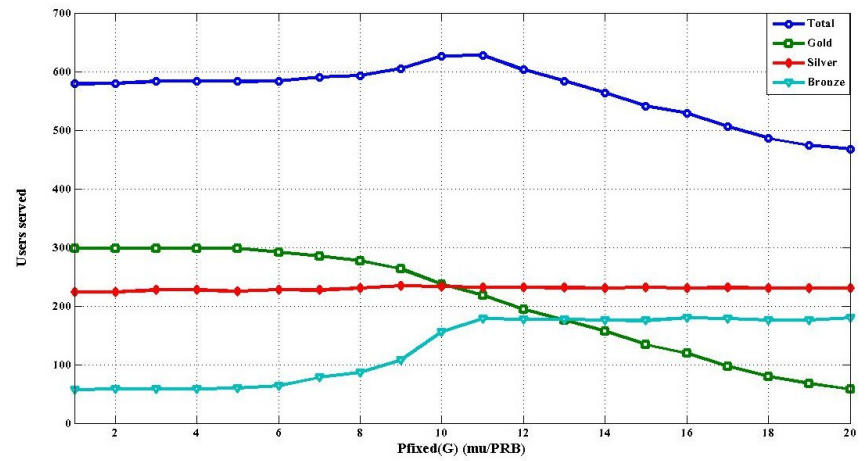

Fig. 2. Mean number of users in the system versus $P_{\text {fixed }}(G)$ when using R2P

$9 \mathrm{mu} / \mathrm{PRB}$, the revenue from the Gold users increases as the price charged increases. When $P_{\text {fixed }}(G)$ exceeds $9 \mathrm{mu} / \mathrm{PRB}$, this revenue decreases because when the price to paid becomes high, Gold users can cancel their connections.

When there are less Gold users in the system, connected Silver users can increase as there are more available PRBs and therefore the additional price for Silver users decreases. Moreover, as the Bronze users are served in the latter, the decrease of connected Gold users offers more free radio resources to Bronze users to connect (see Figure 2). Therefore, we observe that when $P_{\text {fixed }}(G)$ increases, the revenue from Silver and Bronze users increases.

Figure 3 represents the revenue from Gold, Silver, and Bronze users as a function of $P_{\text {fixed }}(S)$. Note that the revenue from Gold users in constant as Gold users are served before Silver users and the price paid by Gold users is independent

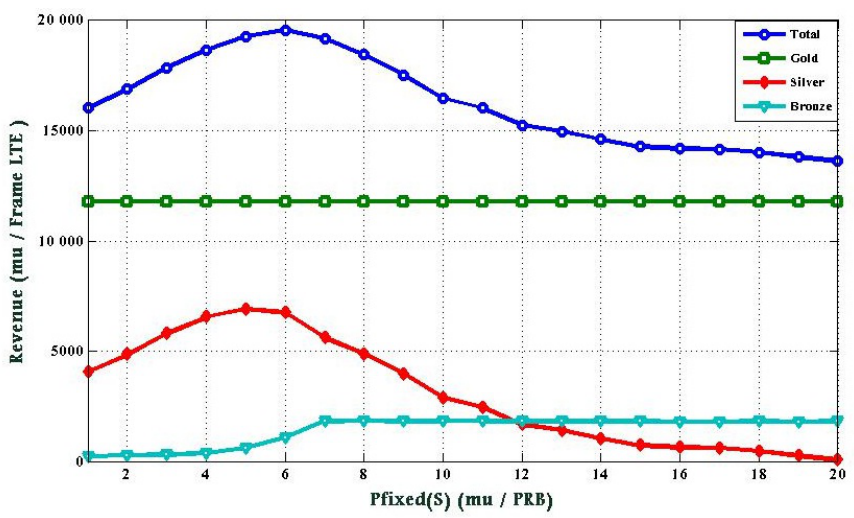

Fig. 3. Mean revenue versus $P_{\text {fixed }}(S)$ when using R2P

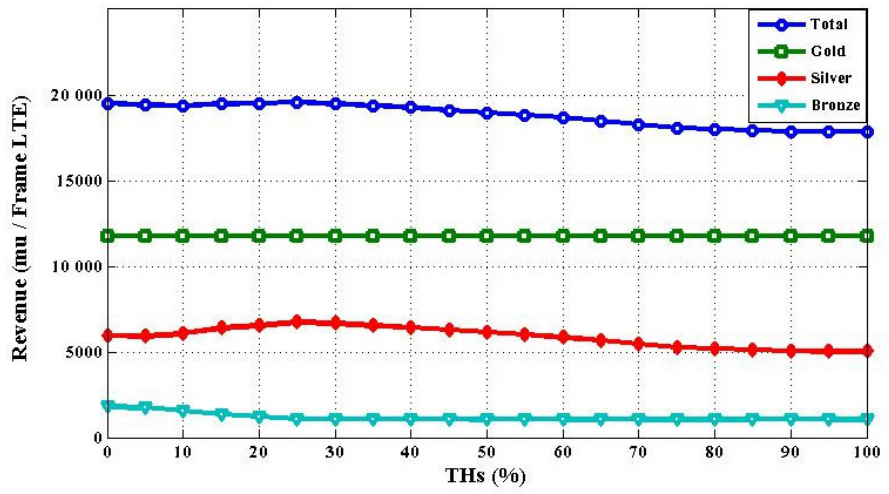

Fig. 4. Mean revenue versus $T H_{s}$

of $P_{\text {fixed }}(S)$. We show that the revenue from Silver users increases then decreases. In fact, the operator revenue depends on the increase of the price to pay and the increase of the blocking rate of Silver users when $P_{\text {fixed }}(S)$ increases.

Figure 4 represents the revenue from Gold, Silver, and Bronze users as a function of $T H_{S}$. When $T H_{S}$ increases, the number of users that do not need to pay additional price increases as congestion periods are delayed. However, the operator does not take advantages from the additional price specially for users having higher valuation. We note that when $T H_{S}$ increases without exceeding $25 \%$, the revenue from Silver users increases as the number of users that pay only a fixed price increases. When $T H_{S}$ exceeds $25 \%$, the operator cannot profit from Silver users that are able to pay higher additional prices.

\section{Pricing schemes comparison}

We now compare between the different pricing schemes. Figure 5 represents the users served ratio when using R2P, SCP, NLP, and FPP. We note FPP almost serves the same ratio for different kinds of users as this pricing scheme uses a fixed price and does not specify any threshold for congestion periods. We also observe that NLP provides the highest ratio of Gold users served as it defines the same congestion threshold for all users categories and favors the Gold users. Therefore, as the radio resources are limited, this pricing scheme serves a very low number of Bronze users. 


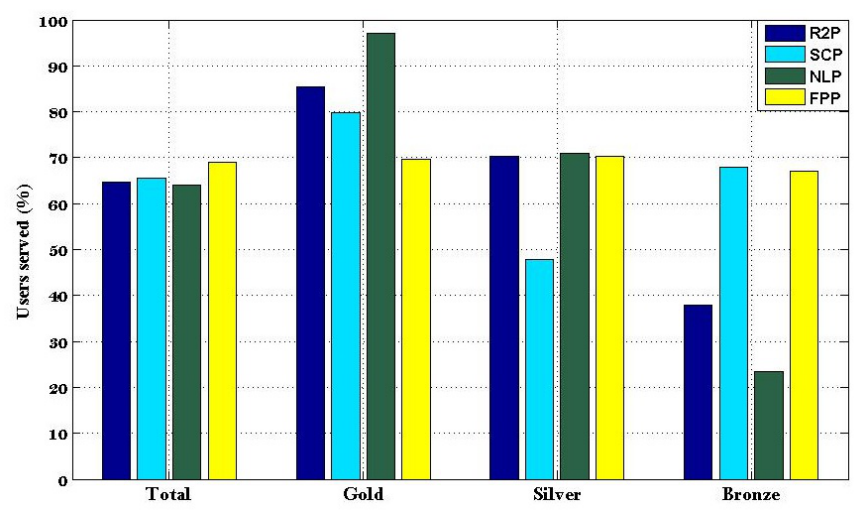

Fig. 5. Users served ratio for different pricing schemes

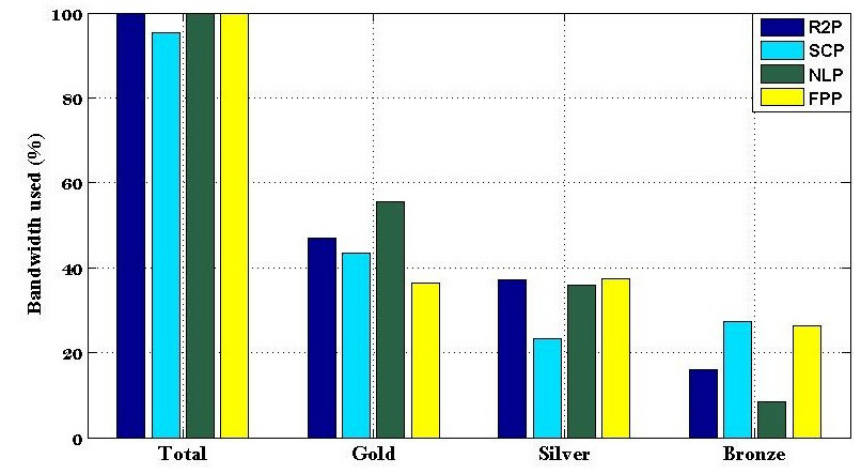

Fig. 6. Bandwidth utilization ratio for different pricing schemes

We note that SCP serves the lowest number of Silver users. In fact, the additional price for this kind of users categories is very high, so many uses prefer to cancel the service connection. As every users category has its own congestion indication threshold, the low number of Silver users served provides more free PRBs for Bronze users that take advantage for low price before a congestion happens. Finally, we show that our proposed pricing scheme gives preference to Gold then Silver and finally Bronze users. Moreover, R2P serves a total number of users near to that served by other pricing schemes. Comparing to SCP and FPP, our pricing scheme does not the highest users served ratio. However, the revenue does not depend only on this performance factor. In fact, an operator has to profit from the willingness-to-pay of users and as every user has its valuation, FPP does not match with a realistic environment. Moreover, a pricing scheme has to use the whole bandwidth. In Figure 6, we show that SCP under-utilizes the bandwidth as it defines a very high additional price. However, we notice that our pricing scheme uses the whole bandwidth and therefore users profit from the total of available bandwidth in order to satisfy their QoS requirements.

Figure 7 represents the mean revenue from different users categories. We note that R2P and SCP outperforms NLP and FPP. In fact, these schemes define a congestion threshold for each user category in order to increase the price to pay when resources become scarce. We also note that $\mathrm{R} 2 \mathrm{P}$ provides the highest revenue. This is due to the radio resource underutilization and the very high additional price when using SCP.

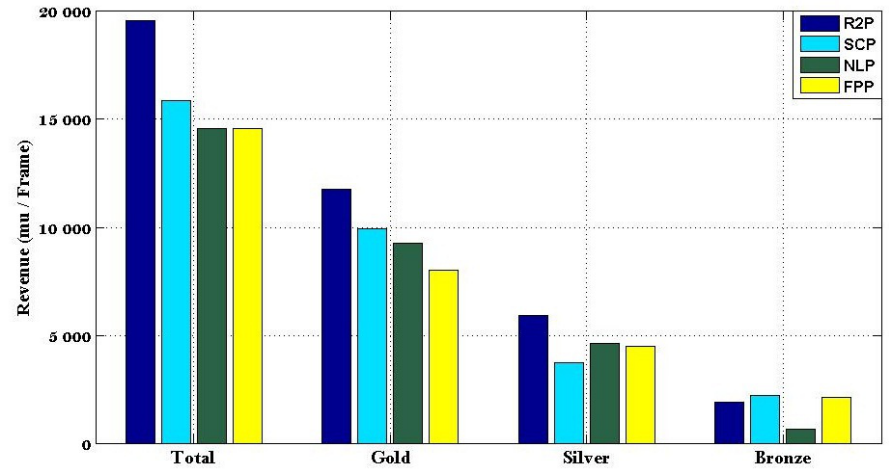

Fig. 7. Operator revenue for different pricing schemes

\section{CONCLUSION}

In this paper, we have evaluated our proposed pricing scheme for different categories of LTE users: Gold, Silver, and Bronze. We compare R2P to various pricing schemes already proposed for LTE as SCP or adapted to this promising technology as FPP and NLP. We show that R2P benefits from the diversity of services and user willingness-to-pay and provides the highest revenue. Moreover, this pricing scheme takes into account the congestion periods and does not totally block users before profiting from the whole bandwidth.

As a part of our future work, we will study the sensitivity [16] of our pricing scheme in order to verify if it still provides the highest revenue even if the operator cannot select the optimal parameters values. We will try to combine this pricing scheme with a sophisticated scheduling algorithm [17] for the 4G LTE-advanced networks using carrier aggregation to improve operator charging.

\section{ACKNOWLEDGMENT}

The authors would like to thank Ahmed Triki and Loutfi Nuaymi for their collaboration.

\section{REFERENCES}

[1] "Realistic LTE Experience: From Peak Rate to Subscriber Experience", White paper, Motorola, August 2009.

[2] "The mobile Broadband Standard Releases" available at http://www.3gpp.org/Releases, last visited in October 2013.

[3] J. Puttonen et al., "Voice-over-IP performance in UTRA long term evolution downlink", IEEE 67th Vehicular Technology Conference, VTC2008Spring, Marina Bay, Singapore, 11 - 14 May 2008.

[4] I. Ahmed and L. Badia, "A quantitative review of the state of the art in quality provisioning for multimedia services over next generation networks", In Proceedings ACM MOBIWAC, Miami Beach, FL, United States, November 2011.

[5] D. Astely et al., "LTE Relase 12 and Beyond", Communications Magazine, IEEE, Volume 51, Issue 7, July 2013.

[6] "LTE: Policy and charging control architecture", 3GPP TS 23.203 version 11.11.0 Release 11, September 2013.

[7] "LTE: Service aspects: Charging and billing", 3GPP TS 22.115 version 11.6.0 Release 11, September 2012.

[8] P. Marbach, "Analysis of a static pricing scheme for priority services", IEEE/ACM Trans. Network, vol. 12, no. 2, pp. 312-325, April 2004.

[9] E. Wallenius and T. Hmlinen, "Pricing model for 3G/4G networks", IEEE PIMRC, Lisbon, pp. 187191, September 2002.

[10] U. Mir and L. Nuaymi, "LTE Pricing Strategies", VTC Spring 2013: Vehicular Technology Conference, Dresde, Germany, June 2013. 
[11] A. Triki and L. Nuaymi, "InterCell Interference Coordination Algorithms in OFDMA wireless systems", IEEE VTC-Spring, Budapest, Hungary, May 2011.

[12] D. Soldani et al., "Strategies for mobile broadband growth: traffic segmentation for better customer experience", IEEE VTC-Spring, Budapest, Hungary, May 2011.

[13] J. Altmann, B. Rupp, and P. Varaiya, "Internet Demand under Different Pricing Schemes", ACM conference on Electronic Commerce, EC-99, Denver, Colorado, USA, 3-5 November 1999.

[14] A. Belghith, L. Nuaymi, and P. Maillé, "Pricing of Real-Time Applications in WiMAX Systems", IEEE 68th Vehicular Technology Conference, VTC2008-Fall, Calgary, Canada, 21-24 September 2008.

[15] A. Belghith, L. Nuaymi, and P. Maillé, "Pricing of differentiated-QoS services WiMAX networks", IEEE Global Communication Conference, IEEE GLOBECOM 2008, New Orleans, LA, USA, 30 November - 4 December 2008.

[16] A. Belghith, L. Nuaymi, X. Lagrange, and P. Maillé, "Sensitivity of a WiMAX pricing strategy", the 20th Personal, Indoor and Mobile Radio Communications Symposium 2009, PIMRC2009, pages 2040 - 2044, Tokyo, Japan, 13 - 16 September 2009.

[17] A. Belghith, "Pricing-based Schedulers for WiMAX", the 5th IEEE International Conference on Wireless and Mobile Computing, Networking and Communications, WiMob2009, pages 202 - 207, Marrakech, Morocco, 12 - 14 October 2009. 